\title{
Angiotensin II modulates cardiovascular autonomic control in the absence of baroreflex loading
}

\author{
J C Vaile, J Fletcher, W A Littler, J H Coote, J N Townend
}

\begin{abstract}
Objective-To investigate the effects of angiotensin II in the absence of baroreflex activation.

Design-Ten healthy male volunteers were studied in a single blind, randomised, crossover study of heart rate variability during intravenous angiotensin II infusion (5-20 ng/kg/min) compared with a control pressor infusion of phenylephrine (0.7$2.8 \mu \mathrm{g} / \mathrm{kg} / \mathrm{min})$. Each infusion was titrated to increase mean blood pressure by $20 \mathrm{~mm} \mathrm{Hg}$; sodium nitroprusside was then infused simultaneously to restore blood pressure to baseline values.

Results-During concomitant angiotensin II (AII) and sodium nitroprusside (SNP) infusion, the mean (SD) RR interval (864 (117) $\mathrm{ms}$ ) was significantly shorter than during phenylephrine (PE) and sodium nitroprusside infusion (1057 (163) $\mathrm{ms})$, and was significantly shorter than at baseline (999 (164) ms), despite comparable levels of blood pressure. Values of high frequency heart rate variability measured in the time and frequency domains were significantly lower during AII/SNP infusion than during PE/SNP: percentage of successive RR interval differences exceeding $50 \mathrm{~ms}, 30(16) \% v 57(21) \%$; root mean square of successive RR interval differences, 63 (39) $v 90$ (40) ms; high frequency power $0.48(0.19) v 0.66(0.26) \mathrm{nu}$.

Conclusions-When the pressor response is controlled by sodium nitroprusside, angiotensin II infusion is associated with tachycardia. Analysis of heart rate variability suggests that this reflects inhibition of cardiac vagal activity.

(Heart 1998;80:127-133)
\end{abstract}

Keywords:angiotensin II; heart rate variability; autonomic nervous system; parasympathetic nervous system

W A Littler

J N Townend

Department of Physiology, University of Birmingham

J Fletcher

$\mathrm{J}$ H Coote

Correspondence to: Dr J C Vaile, Department of Cardiovascular Medicine,

Queen Elizabeth Hospital,

Edgbaston, Birmingham B15

2TH, UK.

email: j.c.vaile@bham.ac.uk

Accepted for publication 24 April 1998 ously suggested that the explanation may the adverse effects of angiotensin II on cardiac autonomic nervous control. ${ }^{67}$
There is strong evidence that impaired cardiac autonomic function is associated with an adverse prognosis after myocardial infarction and in heart failure, ${ }^{89}$ and animal experiments have shown that angiotensin II has direct effects on both vagal and sympathetic nervous control. Inhibition of cardiac vagal activity by circulating angiotensin II is mediated both centrally ${ }^{10}$ and peripherally. ${ }^{11}$ There is also evidence of facilitatory effects on sympathetic nervous activity. ${ }^{12}$ Most of this work has been performed in anaesthetised animals, making it difficult to draw conclusions which can be applied reliably to humans.

Investigation of the effect of angiotensin II on cardiac autonomic activity in intact conscious animals and in humans is hampered by baroreflex activation caused by its powerful pressor effect. One approach has been to control for the pressor effect by comparing the results of angiotensin II infusion with those resulting from a control pressor agent such as phenylephrine. In dogs, little increase in vagal activity occurred during angiotensin II infusion despite the rise in arterial pressure; in contrast there was a marked increase in vagal activity during control pressor infusion of phenylephrine. ${ }^{13}$ In humans, the baroreceptor heart rate reflex is similarly inhibited by angiotensin II. ${ }^{74}$ This does not appear to result from facilitation of sympathetic tone, as tritiated noradrenaline kinetic studies showed no significant difference in sympathetic activity during angiotensin II infusion and during an equipressor infusion of phenylephrine. ${ }^{15}$ In a previous study using analysis of heart rate variability we showed that the primary cause of the reduced heart rate response to pressor doses of angiotensin II was an inhibitory action on cardiac vagal activity. ${ }^{7}$ We postulated that angiotensin II directly inhibits cardiac vagal activity rather than having any specific action on the baroreflex. Such an effect should therefore be demonstrable even in the absence of baroreflex activation.

In this study we investigated the effect of an angiotensin II infusion on cardiac vagal activity in humans while attempting to prevent pressor stimulation of the arterial baroreceptors by simultaneous infusion of the vasodilator sodium nitroprusside. Cardiac vagal activity was determined by analysis of heart rate variability in the time and frequency domains and control experiments were performed using phenylephrine and sodium nitroprusside infusions.

\section{Methods}

Ten male subjects with a mean age of 19 years (range 18 to 23 years) were studied in a single 
Table 1 Blood pressure, RR interval, and RR variability at baseline, before infusion of either drug

\begin{tabular}{lll}
\hline & Phenylephrine & Angiotensin II \\
\hline Bood pressure (mm Hg) & $87(8)$ & $84(8)$ \\
RR interval (ms) & $966(142)$ & $999(164)$ \\
SDNN (ms) & $97(46)$ & $78(32)$ \\
IQDNN (ms) & $118(37)$ & $111(45)$ \\
IQDSD (ms) & $115(57)$ & $117(48)$ \\
RMSSD (ms) & $104(55)$ & $87(43)$ \\
pNN50 (\%) & $59(16)$ & $55(19)$ \\
Total power $\left(\mathrm{ms}^{2}\right)$ & $6996(3449)$ & $8027(5239)$ \\
LF power-abs $\left(\mathrm{ms}^{2}\right)$ & $1269(1090)$ & $1282(1033)$ \\
LF power $(\mathrm{nu})$ & $0.28(0.24)$ & $0.25(0.19)$ \\
HF power-abs $\left(\mathrm{ms}^{2}\right)$ & $3378(2181)$ & $4206(3137)$ \\
HF power $(\mathrm{nu})$ & $0.63(0.21)$ & $0.65(0.17)$ \\
\hline
\end{tabular}

Values are mean (SD).

$\mathrm{p}$ values not significant for any parameter.

abs, absolute units; HF, high frequency; IQDNN, interquartile differences in frequency distributions of the total number of RR intervals; IQDSD, interquartile differences in frequency distributions of successive RR interval differences; LF, low frequency; pNN50, percentage of successive RR interval differences exceeding $50 \mathrm{~ms}$; RMSSD, root mean square of successive RR interval differences; SDNN, standard deviation of RR interval values.

blind crossover study. All subjects were volunteers from staff and students at our institution, all were in good health with no history of cardiovascular or other disease, and all were normotensive (casual supine blood pressure $<140 / 90 \mathrm{~mm} \mathrm{Hg}$ during an initial screening visit). No subject was taking any drugs. The study was approved by the South Birmingham local research ethics committee.

The methods and equipment have been described in detail before. ${ }^{7}$ In brief, subjects were asked not to eat or drink for at least two hours before the experiments, which were performed in a dedicated clinical autonomic research room at $21-24^{\circ} \mathrm{C}$. Two venous cannulas were inserted into unilateral forearm veins for drug administration. Skin electrodes were applied to the chest wall for electrocardiographic monitoring. The electrocardiographic signal was amplified, processed (high frequency signal noise filter $>500 \mathrm{~Hz}$ ), and digitised at $165 \mathrm{~Hz}$ for each channel using a National Instruments NB/M10/16XH/18 analogue to digital converter board (National Instruments Corporation, Austin, Texas, USA). $\mathrm{R}$ waves were detected by individually adjusted thresholds and a maximum to minimum voltage difference within five samples $(0.04 \mathrm{~s})$ of $>0.5 \mathrm{~V}$. The signal was displayed on the screen of a personal computer running Lab View 3.1 software (National Instruments Corporation) and selected periods were stored to disk. Blood pressure was continuously monitored from the index finger using the Finapres device (Ohmeda 2300, Liberty Corner, New Jersey, USA) and from intermittent conventional sphygmomanometry. Subjects were studied in the semisupine position, and respiration was maintained at a constant rate in each arm of the study by asking subjects to synchronise their respiration to an audio signal generator, which during the first visit was adjusted to suit each individual's respiratory frequency. A respiratory signal was obtained, displayed, and recorded from two electrodes attached to the chest wall to monitor changes in impedance with respiration.
After a minimum of 30 minutes' rest to achieve a stable heart rate (ensuring that mean rate over two 30 second recordings five minutes apart varied by $<10 \%$ ) the baseline electrocardiographic recording was acquired, consisting of at least 256 consecutive RR intervals.

Subjects were randomly assigned to angiotensin II or a control infusion of phenylephrine during the first of two studies; the other agent was given during a second study, seven to 14 days later. Angiotensin II analogue (Hypertensin, Ciba Geigy Pharmaceuticals, Horsham, West Sussex, UK) was infused in normal saline at rates of 5 to $20 \mathrm{ng} / \mathrm{kg} / \mathrm{min}$. Phenylephrine was infused at rates of 0.7 to $2.8 \mu \mathrm{g} / \mathrm{kg} / \mathrm{min}$. Infusion rates were increased incrementally until a rise in mean blood pressure of 10 to $20 \mathrm{~mm} \mathrm{Hg}$ was achieved. After a 10 minute period to allow equilibration, a recording of at least 256 consecutive RR intervals was taken. An infusion of sodium nitroprusside was then started, beginning at a rate of $1 \mathrm{mg} /$ hour, and the dose was titrated to achieve restoration (to within $5 \mathrm{~mm} \mathrm{Hg}$ ) of the subjects' baseline mean arterial pressure. The maximum dose used was $12 \mathrm{mg} /$ hour. A further period of ECG recording (256 RR intervals) was then taken.

\section{DATA ANALYSIS}

All ECG series were reviewed and if necessary edited before analysis to exclude ectopic and artefact signals. No signal contained more than $1 \%$ of ectopic beats; when ectopic beats were deleted the RR interval was replaced with a running mean. The ECG series for analysis were coded so that the investigator performing the analysis was blinded to the vasoactive agent or agents under study. Heart rate variability was analysed off line using both the Lab-View 3.0 software and Statview (Abacus Concepts Inc, San Francisco, California, USA). We used the standard time domain measures of SDNN (standard deviation of RR interval values), RMSSD (root mean square of successive RR interval differences), and pNN50 (percentage of successive RR interval differences exceeding $50 \mathrm{~ms}$ ). In addition we determined the interquartile differences ( 75 th to 25 th centile) of the frequency distributions of the total number of RR intervals (IQDNN) and of successive RR interval differences (IQDSD). As we have reported before, ${ }^{7}$ IQDNN and IQDSD are simple indices, which exclude extreme values at each end of the frequency distribution and minimise the influence of any artefact or ectopic activity that may have escaped the editing process.

Frequency domain analysis was performed to determine the power of the underlying component oscillations. Stationarity of the time series was tested by calculation of the mean and variance of the first and last 128 beats of each recording period in order to verify a difference of $<10 \%$ in the values for each time series. The mean was subtracted from each point in the RR interval series, and power spectral analysis was performed using the Burg algorithm ${ }^{16}$ with a model order between 8 and $12 .{ }^{17}$ The power of each underlying frequency 
Table 2 Blood pressure, RR interval, and RR variability during phenylephrine or angiotensin II infusion

\begin{tabular}{llll}
\hline & Phenylephrine & Angiotensin II & p value \\
\hline Blood pressure (mm Hg) & $110(8)$ & $107(4)$ & NS \\
RR interval (ms) & $1364(201)$ & $1019(159)$ & $<0.001$ \\
SDNN (ms) & $79(29)$ & $114(66)$ & NS \\
IQDNN (ms) & $138(119)$ & $162(145)$ & NS \\
IQDSD (ms) & $153(93)$ & $152(153)$ & NS \\
RMSSD (ms) & $89(50)$ & $122(114)$ & NS \\
pNN50 (\%) & $54(23)$ & $57(16)$ & NS \\
Total power $\left(\mathrm{ms}^{2}\right)$ & $11674(13800)$ & $17902(20511)$ & NS \\
LF power-abs $\left(\mathrm{ms}^{2}\right)$ & $685(456)$ & $2326(2229)$ & 0.008 \\
LF power $(n u)$ & $0.17(0.13)$ & $0.23(0.11)$ & NS \\
HF power-abs $\left(\mathrm{ms}^{2}\right)$ & $5502(6959)$ & $8673(14167)$ & NS \\
HF power $(\mathrm{nu})$ & $0.56(0.26)$ & $0.60(0.19)$ & NS \\
\hline
\end{tabular}

Values are mean (SD).

abs, absolute units; HF, high frequency; IQDNN, interquartile differences in frequency distributions of the total number of RR intervals; IQDSD, interquartile differences in frequency distributions of successive RR interval differences ; LF, low frequency; pNN50, percentage of successive RR interval differences exceeding $50 \mathrm{~ms}$; RMSSD, root mean square of successive RR interval differences; SDNN, standard deviation of RR interval values.

was quantified by decomposing the total variability signal with the method of Zetterberg. ${ }^{18}$ This enables the determination of low frequency (LF) power at $\sim 0.1 \mathrm{~Hz}$ (reflecting both sympathetic and vagal activity ${ }^{19}$ ) and high frequency (HF) power at the measured respiratory frequency. Because total power varies greatly between individual subjects, power was determined in both absolute units and as normalised values. The power in normalised units was calculated by dividing the absolute power of a given component (area under the component curve) by the total power minus the 0-0.04 Hz component.

STATISTICAL ANALYSIS

Data for blood pressure, RR interval, and RR interval variability were tested for normality. The significance of the differences between groups was determined using Student's $t$ test for normally distributed data; otherwise the Wilcoxon signed rank test was used.

\section{Results}

RESULTS AT BASELINE

Baseline values of mean blood pressure, RR interval, and measures of heart variability in

Subject (a)

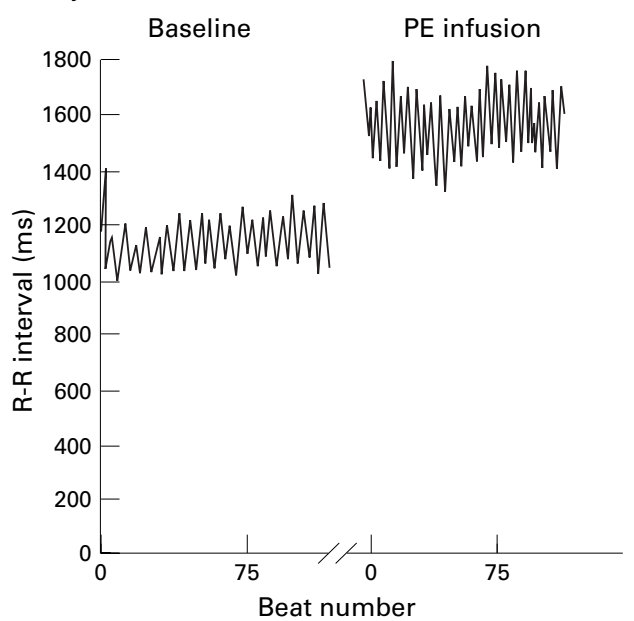

both time and frequency domains were not significantly different when subjects received angiotensin II or phenylephrine (table 1).

RESULTS DURING PRESSOR INFUSIONS

During the pressor infusions of angiotensin II and phenylephrine, although the mean level of induced hypertension was equal, the pulse interval responses were widely divergent; during angiotensin II infusion the mean RR interval was significantly shorter than with phenylephrine $(\mathrm{p}<0.001)$.

The group mean measures of heart rate variability were not significantly different (with the exception of LF absolute power) during infusion of angiotensin II or phenylephrine (table 2), despite the widely divergent heart rate responses. The marked bradycardia associated with phenylephrine infusion was not accompanied by a significant increase in measures of cardiac vagal activity. It was apparent that these mean data comprised two different heart rate variability responses to pressor infusions of phenylephrine. These are illustrated by the tachograms in fig 1 . In the first example (subject a), phasic variation in heart rate (respiratory sinus arrhythmia) is increased during phenylephrine infusion, a response which can be interpreted as an increase in cardiac vagal activity. However, in subject $b$, although there is a bradycardia, heart rate variability is severely reduced. The latter paradoxical response occurred in five of the 10 subjects.

\section{RESULTS AFTER COUNTERACTION OF THE}

PRESSOR EFFECT

Following near restoration of baseline arterial pressure by concomitant infusion of sodium nitroprusside we were able to compare measures of heart rate variability without the confounding influences of baroreflex loading. The doses of sodium nitroprusside required to counteract the pressor effects of angiotensin II and phenylephrine (mean (SD)) were not significantly different at 6.1 (1.5) and 6.9

Subject (b)

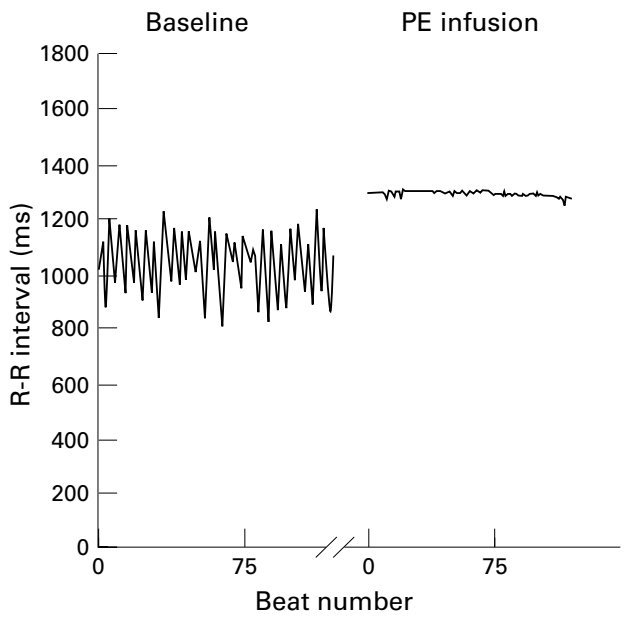

Figure 1 Typical examples of the two different heart rate variability responses to pressor infusions of phenylephrine, illustrated by the tachograms taken from two individual subjects, at baseline and during a pressor infusion of phenylephrine. In subject a phasic variation in heart rate (respiratory sinus arrhythmia) is increased during phenylephrine infusion, a response which can be interpreted as an increase in cardiac vagal activity. In subject $b$, although there is a bradycardia, heart rate variability is severely reduced. 
Table 3 Blood pressure, $R R$ interval, and $R R$ variability during infusion of phenylephrine or angiotensin II, each with sodium nitroprusside

\begin{tabular}{llll}
\hline & Phenylephrine & Angiotensin II & p value \\
\hline Blood pressure (mm Hg) & $91(12)$ & $88(9)$ & NS \\
RR interval (ms) & $1057(163)$ & $864(117)$ & $<0.001$ \\
SDNN (ms) & $93(25)$ & $86(35)$ & NS \\
IQDNN (ms) & $178(94)$ & $129(62)$ & NS \\
IQDSD (ms) & $126(59)$ & $65(42)$ & 0.004 \\
RMSSD (ms) & $90(40)$ & $63(39)$ & 0.04 \\
pNN50 (\%) & $57(21)$ & $30(16)$ & $<0.001$ \\
Total power (ms $\left.{ }^{2}\right)$ & $16361(13341)$ & $10776(9819)$ & NS \\
LF power-abs $\left(\mathrm{ms}^{2}\right)$ & $930(829)$ & $1748(1288)$ & $<0.05$ \\
LF power $(\mathrm{nu})$ & $0.15(0.11)$ & $0.45(0.21)$ & 0.005 \\
HF power-abs $\left(\mathrm{ms}^{2}\right)$ & $4717(3672)$ & $2763(4201)$ & NS \\
HF power $(\mathrm{nu})$ & $0.66(0.26)$ & $0.48(0.19)$ & $<0.05$ \\
\hline
\end{tabular}

Values are mean (SD).

abs, absolute units; HF, high frequency; IQDNN, interquartile differences in frequency distributions of the total number of RR intervals; IQDSD, interquartile differences in frequency distributions of successive RR interval differences; LF, low frequency; pNN50, percentage of successive $\mathrm{RR}$ interval differences exceeding $50 \mathrm{~ms}$; RMSSD, root mean square of successive RR interval differences; SDNN, standard deviation of RR interval values.

(2.1) $\mathrm{mg} /$ hour, respectively. The most striking result was that the heart rate changes in response to the two pressor agents remained divergent, with a shorter mean RR interval during infusion of angiotensin II and sodium nitroprusside (AII/SNP) than during phenylephrine and sodium nitroprusside (PE/SNP). Compared with baseline, AII/SNP resulted in a slight tachycardia $(p<0.02)$ while there was no significant difference in heart rate from baseline during PE/SNP (table 3, fig 2). The heart rate variability responses to the two infusions are given in table 3 . There was no significant difference in time domain measures of overall RR variability such as SDNN or
IQDNN. However, measures of high frequency variation such as IQDSD, RMSSD, and pNN50 were significantly greater during $\mathrm{PE} /$ SNP than during AII/SNP infusions. Using frequency domain analysis, total power was not significantly different. There was a trend to greater absolute values for $\mathrm{HF}$ power and lower values for LF power during PE/SNP than during AII/SNP. When interpatient variance was reduced by the use of normalised units there was significantly greater high frequency power and lesser low frequency power during PE/ SNP than during AII/SNP. Figure 2 shows the heart rate variability of an individual subject at baseline, and the responses to phenylephrine and angiotensin II infusions, during pressor infusion, and during correction for the increase in arterial pressure with sodium nitroprusside. In this subject, variability of successive differences between $R R$ intervals increased in response to phenylephrine but did not change with angiotensin II. When the arterial pressure was returned to control level, the variability was returned toward baseline with phenylephrine and reduced to a value lower than baseline with angiotensin II.

\section{Discussion}

MEASUREMENT OF CARDIAC VAGAL ACTIVITY IN MAN

The vagus nerve is not accessible to recording techniques in man, so measurement of cardiac vagal efferent activity is necessarily indirect. Heart rate variability measures of vagal activity
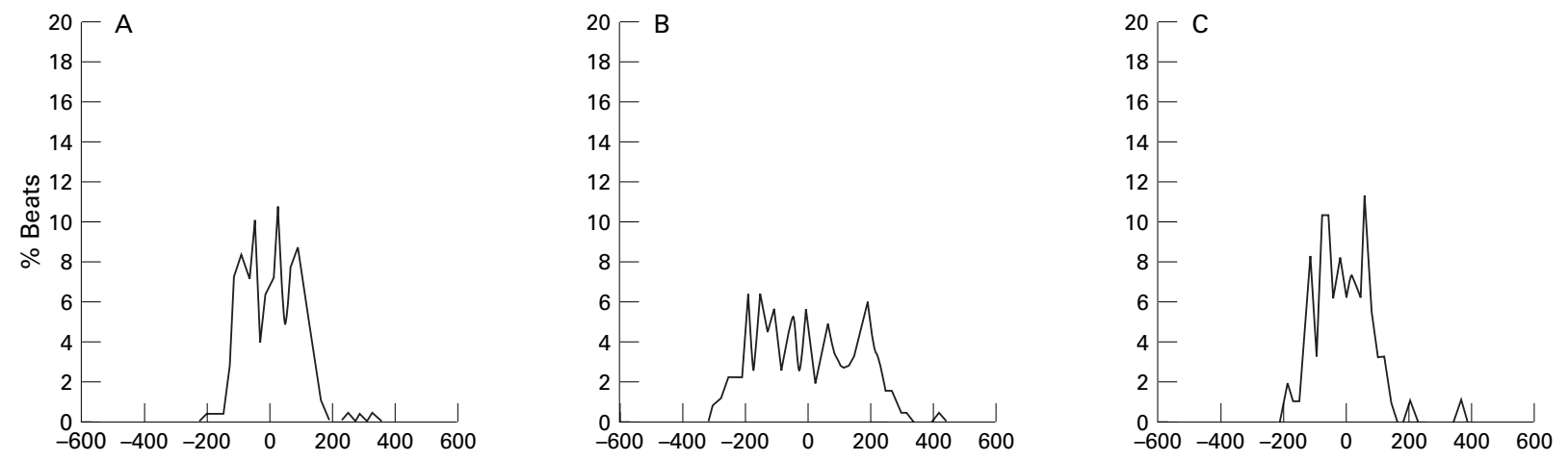

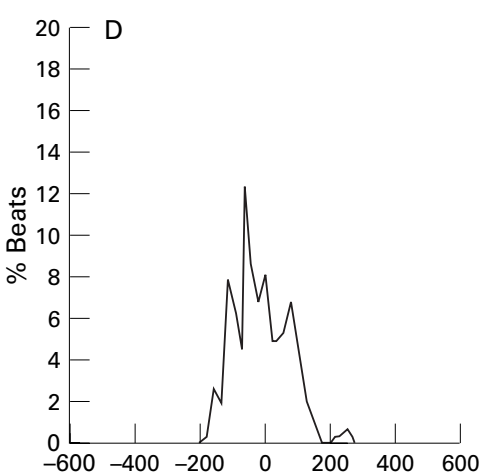

$\mathrm{R}-\mathrm{R}$ interval successive difference ( $\mathrm{ms}$ )

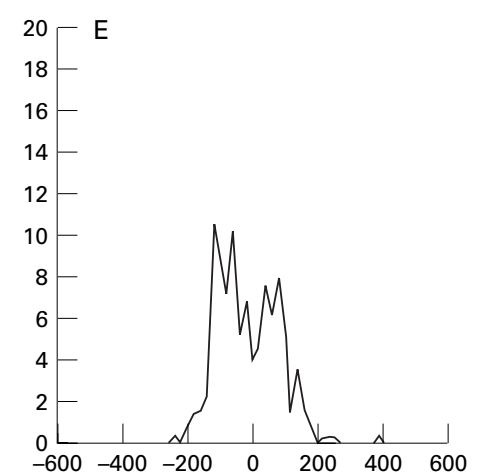

$\mathrm{R}-\mathrm{R}$ interval successive difference (ms)

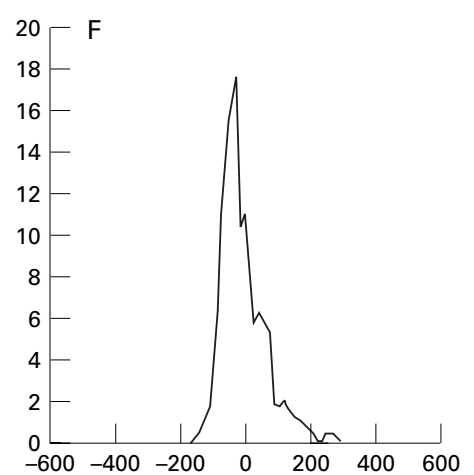

$\mathrm{R}-\mathrm{R}$ interval successive difference ( $\mathrm{ms}$ )

Figure 2 Results of an individual subject are shown using frequency histograms of successive RR interval differences. Plots $(A)$ and (D) show beat to beat variability at baseline. During pressor infusions, variability increased in response to phenylephrine (B) but did not change with angiotensin II (E). When the arterial pressure was returned to control level by simultaneous infusion of sodium nitroprusside, the variability was returned to baseline with phenylephrine $(C)$ and reduced to below baseline with angiotensin II $(F)$. 
assess the high frequency ("beat to beat") variability resulting from vagal modulation of heart rate caused by respiratory sinus arrhythmia. ${ }^{19-21}$ This high frequency variability is specifically measured by indices such as the variability of successive intervals between $R R$ intervals (IQDSD), RMSSD, and pNN50 rather than by measures of total variability such as SDNN. ${ }^{22}$

Analysis of heart rate variability in the frequency domain allows determination of the power and frequency of component oscillations. The magnitude of respiratory modulation of heart rate can be measured by determining the power of the component which is synchronous with respiration. This is usually centred at about $0.25 \mathrm{~Hz}$ and provides a further index of cardiac vagal tone. ${ }^{192}$

No measure of heart rate variability provides an absolute measure of the activity of vagal (or indeed sympathetic) nervous activity. These measurements do, however, reflect the degree of neural modulation of heart rate.

HEART RATE VARIABILITY RESPONSES TO PRESSOR INFUSIONS

In a previous paper we showed that during a pressor infusion of intravenous angiotensin II, the baroreflex mediated increase in cardiac vagal activity assessed by heart rate variability was significantly lower than during a control infusion of phenylephrine. ${ }^{7}$ This confirmed the results of earlier animal studies which were able to use direct neural recording to show inhibition of vagal activity during baroreflex loading. ${ }^{13}$ The results arising from the first part of this study are at first sight discrepant, with no significant difference between measures of heart rate variability (with the sole exception of $\mathrm{LF}$ absolute power) during pressor infusions of angiotensin II and phenylephrine. We believe that the reason for this lies in the limitation of heart variability as a measure of cardiac vagal activity during extremes of baroreflex loading. In the present study the mean arterial pressure was $110 \mathrm{~mm} \mathrm{Hg}$ during phenylephrine infusion, while in our previous study the equivalent value was lower at $104 \mathrm{~mm} \mathrm{Hg.}{ }^{7}$ In a study examining the effect of graded stimulation of vagal activity using a pressor infusion of phenylephrine. Goldberger et al found that there is often a paradoxical reduction in measures of high frequency variability (caused by respiratory sinus arrhythmia) with high levels of vagal stimulation, ${ }^{23}$ a finding we have confirmed (fig 1 , subject $b$ ). There are several possible explanations for this lack of consistent relation between vagal activity and heart rate variability. Potential mechanisms include:

(1) Loss of respiratory phasic variation in vagal activity at high blood pressures ${ }^{24}$ caused by a central effect on cardiac vagal motor neurone activity. It is possible that at levels of high excitation owing to baroreflex loading the neurones might become unresponsive to either further excitatory stimuli or inhibitory stimuli related to respiratory activity. Thus respiratory variation will be lost.
(2) Alternatively, phasic discharge of vagal efferent activity may persist with a loss of effect at the sinus node. Saturation of the acetylcholine dose-response curve has been postulated $\mathrm{d}^{25}$ or there may be a minimum rate of sinus node discharge at which point variability owing to further slowing cannot occur.

HEART RATE VARIABILITY RESPONSES AFTER COUNTERACTION OF THE PRESSOR EFFECT

The major advantage of our study design was that the limitations imposed by baroreflex activation were overcome when the pressor effect of phenylephrine or angiotensin II was controlled by concomitant vasodilator infusion. Without simultaneous vagal stimulation from baroreflex loading, heart rate variability is able to reflect more subtle changes in efferent cardiac vagal activity. The results shown in table 3 appear to indicate that cardiac vagal efferent activity is indeed inhibited by circulating angiotensin II in the absence of baroreflex loading. The most notable feature of this effect is an increase in resting heart rate with angiotensin II.

The low frequency power component $(0.1 \mathrm{~Hz})$ has been used as an index of sympathetic activity, although there is undoubtedly a large vagal component to this oscillation. ${ }^{19}$ The power of this component was higher during angiotensin II infusion and we are unable to exclude facilitation by angiotensin II of sympathetic nervous activity in addition to inhibition of vagal activity.

SITE OF ACTION OF ANGIOTENSIN II

Animal experiments suggest that angiotensin II exerts both peripheral ${ }^{11}$ and central ${ }^{13}$ inhibitory actions on vagal activity. The central actions appear to be mediated through the circumventricular organs such as the area postrema and subfornical organ, which contain angiotensin II sensitive neurones and which, lacking a bloodbrain barrier, are accessible to circulating angiotensin II. ${ }^{10}$ In a series of papers examining the influence of angiotensin II on the control of human sympathetic activity, Goldsmith and colleagues were unable to confirm animal work suggesting tonic central and peripheral presynaptic facilitation of sympathetic activity. ${ }^{1526}$ However, they did show that the normal reduction in forearm vascular resistance during baroreflex loading was attenuated by angiotensin II, suggesting that the effects of angiotensin II on sympathetic activity in humans are limited to inhibition of baroreflex mediated changes. ${ }^{27}$ This does not appear to be the case for cardiac vagal activity, which we have shown to be attenuated even in the absence of baroreflex loading.

Although inhibition of efferent cardiac vagal activity is supported by animal evidence, it is possible that differences in the afferent limb of the baroreflex contributed to our results. We controlled for mean blood pressure because this is the main determinant of baroreceptor discharge, with relatively little effect from other variables (for example, pulse pressure, rate of pressure change). ${ }^{28}$ However, we cannot ex- 
clude influences from these variables merely because mean blood pressure was unchanged. Equally, it is possible that angiotensin II and phenylephrine exert different actions on the mechanical properties of the baroreceptor. Phenylephrine has been shown to cause vasoconstriction of the carotid sinus in dogs. ${ }^{29}$ In rabbits, angiotensin II increased the dimensions of the aortic arch, while phenylephrine resulted in vasoconstriction although there were no significant differences in aortic nerve activity. ${ }^{30}$ Lumbers et al also found no significant differences between the effects of angiotensin II and phenylephrine on afferent baroreceptor activity. ${ }^{13}$ We therefore believe that an afferent effect is unlikely to account for the results of our study.

\section{LIMITATIONS}

Modulation of autonomic activity by nitric oxide as suggested by recent animal evidence may have exerted a confounding influence on this study. This information was not available at the time our study was designed so the nitric oxide donor sodium nitroprusside rather than a non-endothelium dependent vasodilator was used to counteract the pressor effects of angiotensin II and phenylephrine. Nitric oxide synthase (NOS) is found at both central and peripheral sites involved in the integration of the arterial baroreflex and inhibition of NOS causes an increase in baroreflex sensitivity in conscious rabbits. ${ }^{31}$ In the ferret, the bradycardia resulting from vagal stimulation is attenuated by NOS inhibition suggesting that efferent vagal activity is modulated by NO. ${ }^{32}$ The effects of nitric oxide on autonomic control in humans are unknown and require further investigation, but a small study of the effects of NO inhibition found no evidence of modulation of autonomic activity. ${ }^{33} 34$ In our study the lack of any significant difference in dose of sodium nitroprusside between the phenylephrine and angiotensin II groups makes it unlikely that the differences in high frequency heart rate variability measures were the result of modulation of vagal activity by NO from sodium nitroprusside.

A second limitation is that we used a sampling rate of $165 \mathrm{~Hz}$, which may have resulted in an error of up to $12 \mathrm{~ms}$ per RR interval. However, it would not have introduced any bias and differences in high frequency heart rate variability were demonstrable despite this technical limitation.

\section{CLINICAL IMPLICATIONS}

Our results may have important clinical implications for the underlying mechanisms of action of drugs inhibiting the reninangiotensin system including angiotensin converting enzyme (ACE) inhibitors and angiotensin II receptor antagonists. Low cardiac vagal activity measured by heart rate variability and baroreflex sensitivity has been associated with an adverse prognosis after myocardial infarction $^{835}$ and in heart failure. ${ }^{9}{ }^{36}$ Treatment with ACE inhibitors has been shown to increase heart rate variability and baroreflex sensitivity in both groups of patients, ${ }^{37-39}$ suggesting that at least part of the cause of reduced cardiac vagal activity is the increase in circulating angiotensin II found in these conditions. The reasons why ACE inhibitors reduce mortality in heart failure and after myocardial infarction ${ }^{240}$ are not understood but we suggest that an increase in cardiac vagal activity as a result of a reduction in circulating angiotensin II may be an important component mechanism. Cardiac vagal activity appears to exert an antiarrhythmic action in experimental animals by increasing the fibrillatory threshold of ischaemic myocardium. ${ }^{41}$ If an increase in vagal activity associated with ACE inhibitor treatment acts in humans to reduce ventricular arrhythmias and fibrillation, a reduction in mortality from sudden presumed arrhythmic death might be expected. There is now abundant evidence for such an effect from the results of trials of ACE inhibitors in both heart failure ${ }^{45}$ and myocardial infarction. ${ }^{142}$ In view of the results of the ELITE trial, ${ }^{3}$ studies investigating the effects of angiotensin II receptor blockade on cardiac autonomic control are required.

This study was supported by the British Heart Foundation.

1 Kober L, Torp-Pedersen C, Carlsen JE, et al. A clinical trial of the angiotensin converting enzyme inhibitor trandolapril in patients with left ventricular dysfunction after myocardial infarction. $N$ Engl f Med 1995;333:1670-6.

2 ISIS-4 Collaborative Group. A randomised trial assessing early oral captopril, oral mononitrate and intravenous magnesium sulphate in 58050 patients with suspected acute myocardial infarction. Lancet 1995;345:669-85.

3 Pitt B, Segal R, Martinez FA, et al. Randomised trial of losartan versus captopril in patients over 65 with heart failure (ELITE). Lancet 1997;349:747-52.

4 Cohn J, Johnson G, Ziesche S, et al. A comparison of enalapril with hydralazine-isosorbide dinitrate in the treatment of chronic congestive heart failure. $N$ Engl F Med 1991;325: 303-10.

5 Fonarow G, Chelimsky-Fallick C, Warner Stevenson L, et al. Effect of direct vasodilatation with hydralazine versus angiotensin-converting enzyme inhibition with captopril on mortality in advanced heart failure: the $\mathrm{Hy}-\mathrm{C}$ trial. $f \mathrm{Am}$ Coll Cardiol 1992;19:842-50.

6 Townend JN, Littler WA. Cardiac vagal activity: a target for intervention in heart disease. Lancet 1995;345:937-8.

7 Townend JN, Al-Ani M, West JN, et al. Modulation of cardiac autonomic control in humans by angiotensin II. Hypertension 1995;25:1270-5.

8 La Rovere MT, Specchia G, Schwartz PJ. Baroreflex sensitivity, clinical correlates and cardiovascular mortality sensitivity, clinical correlates and cardiovascular mortality among patients with
tion 1988;78:816-24.

9 Binder T, Frey B, Porenta G, et al. Prognostic value of heart rate variability in patients awaiting cardiac transplantation. PACE 1992;15:2215-20.

10 Joy M, Lowe R. Evidence that the area postrema mediates the central cardiovascular response to angiotensin II. Clin Sci 1970;41:89-100.

11 Potter EK. Angiotensin inhibits action of vagus nerve at the heart. Br F Pharmacol 1982;75:9-11.

12 Zimmerman BG. Adrenergic facilitation by angiotensin: does it serve a physiological function? Clin Sci 1981;60: 343-8.

13 Lumbers E, McCloskey D, Potter E. Inhibition by angiotensin II of baroreceptor-evoked activity in cardiac vagal efferent nerves in the dog. $\mathcal{F}$ Physiol (Lond) 1979;294: vagal ef 69 .

14 Mace PJ, Watson RD, Skan W, et al. Inhibition of the baroreceptor heart rate reflex by angiotensin II in normal man. Cardiovasc Res 1985;19:525-7.

15 Goldsmith S, Hasking G. Effect of a pressor infusion of angiotensin II on sympathetic activity and heart rate in normal humans. Circ Res 1991;68:263-8.

16 Burg JP. A new analysis technique for time series data. Erschede: NATO Advanced Study Institute on Signal Processing with emphasis on underwater acoustics, 1968.

17 Akaike H. Statistical predictor identification. Ann Int Stat Math 1970;22:203-17.

18 Zetterberg LH. Estimation of parameters for a linear difference equation with application to EEG analysis. Math Biosci 1969;5:227-75.

19 Pagani M, Lombardi F, Guzzett S, et al. Power spectral analysis of heart rate and arterial pressure variabilities as a marker of sympatho-vagal interaction in man and conscious dog. Circ Res 1986;59:178-93. 
20 Katona PG, Jih F. Respiratory sinus arrhythmia: noninvasive measure of parasympathetic cardiac control. $\mathcal{F}$ Appl Physiol $1975 \cdot 39 \cdot 801-5$.

21 Akselrod S, Gordon D, Ubel FA, et al. Power spectrum analysis of heat rate fluctuations: a quantitative probe of 2.

22 Task Force of the European Society of Cardiology and the North American Society of Pacing and Electrophysiology. Heart rate variability. Standards of measurement, physiological interpretation, and clinical use. Circulation 1996; 93:1043-65.

23 Goldberger JJ, Kim YH, Ahmed MW, et al. Effect of graded increases in parasympathetic tone on heart rate variability. 7 Cardiovasc Electrophysiol 1996;7:594-602.

24 Kunze D. Reflex discharge patterns of cardiac vagal efferent fibres. F Physiol (Lond) 1972;222:1-15.

25 Malik M, Camm AJ. Components of heart rate variabilitywhat they really mean and what we really measure. $A m \mathcal{F}$ what they really mean
Cardiol $1993 ; 72: 821-2$.

26 Goldsmith S, Hasking G. Subpressor angiotensin II infusions do not stimulate sympathetic activity in humans. infusions do not stimulate sympa

27 Goldsmith S, Hasking G, Miller E. Angiotensin II inhibits the forearm vascular response to increased arterial pressure in humans. F Am Coll Cardiol 1995;25:246-50.

28 Schmidt R, Kumada M, Sagawa K. Cardiovascular responses to various pulsatile pressures in the carotid sinus. Am F Physiol 1972;223:1-7.

29 Peveler RC, Bergel DH, Robinson JL, et al. The effect of phenylephrine upon arterial pressure, carotid sinus radius and baroreflex sensitivity in the conscious greyhound. Clin Sci 1983;64:455-61.

30 Hirooka Y, Imaizumi T, Sugimachi M, et al. Mechanisms involved in aortic baroreceptor excitation during druginduced aortic pressure elevation in intact rabbits. $\mathcal{F}$ Auton Nerv Sys 1992;40:99-106.

31 Liu JL, Murakami H, Zucker IH. Effects of NO on baroreflex control of heart rate and renal nerve activity in conscious rabbits. Am f Physiol 1996;270:R1361-70.
32 Conlon K, Collins T, Kidd C. Modulation of vagal actions on heart rate produced by inhibition of nitric oxide synthase in the anaesthetised ferret. Exp Physiol 1996;81: 547-50.

33 Hansen J, Jacobsen TN, Victor RG. Is nitric oxide involved in the tonic inhibition of central sympathetic outflow in humans? Hypertension 1994;24:439-44.

34 Castellano M, Rizzoni D, Beschi M, et al. Relationship between sympathetic nervous system activity, baroreflex and cardiovascular effects after acute nitric oxide synthesis inhibition in humans. F Hypertens 1995;13:1153-61.

35 Kleiger RE, Miller JP, Bigger JT, et al. Decreased heart rate variability and its association with increased mortality after acute myocardial infarction. Am f Cardiol 1987;59:256-62.

36 Osterziel KJ, Hanlein D, Willenbrock R, et al. Baroreflex sensitivity and cardiovascular mortality in patients with mild to moderate heart failure. Br Heart f 1995;73:517-22.

37 Townend JN, West JN, Davies $M$, et al. Effect of quinapril on blood pressure and heart rate in congestive heart failure. Am f Cardiol 1992;69:1587-90.

38 Binkley P, Haas G, Starling R, et al. Sustained augmentation of parasympathetic tone with angiotensin-converting enzyme inhibition in patients with congestive heart failure. $\mathcal{F}$ Am Coll Cardiol 1993;21:655-61.

39 Bonaduce D, Marciano F, Petretta M, et al. Effects of converting enzyme inhibition on heart period variability in patients with acute myocardial infarction. Circulation 1994 ; 90:108-13.

40 SOLVD investigators. Effect of enalapril on survival in patients with reduced left ventricular ejection fractions and congestive heart failure. N Engl f Med 1991;325:293-302.

41 Vanoli E, De Ferrari GM, Stramba-Badiale M, et al. Vagal stimulation and prevention of sudden death in conscious dogs with a healed myocardial infarction. Circ Res 1991;68: 1471-81.

42 GISSI-3. Effects of lisinopril and transdermal glyceryl trinitrate singly and together on 6 week mortality and ventricular function. Lancet 1994;343:1115-22. 